\title{
Re: Long-Term Outcome of Low Scrotal Approach Orchiopexy without Ligation of the Processus Vaginalis
}

\author{
Hyuga T, Kawai S, Nakamura S, Kubo T, Nakai H \\ Jichi Medical University, Children's Medical Center Tochigi, Department of Pediatric Urology, Tochigi, Japan
}

J Urol 2016;196:542-547. doi: 10.1016/j.juro.2016.02.2962. Epub 2016 Mar 2.

\section{EDITORIAL COMMENT}

Dealing with pre-scrotal cryptorchidism is a common practice for pediatric urologists. Surgical correction is the standard care for these children. There are several surgical techniques which can be performed with no definitive long-term results presented in the literature. In general practice, ligation of patent processus vaginalis (PV) is routinely performed during orchiopexy to avoid an inguinal hernia. In this retrospective study, the authors present their long-term outcomes for patients who underwent low scrotal approach orchiopexy without PV ligation. Intraoperative findings show 10 testes with widely patent PV and not widely patent in 217 testes. The authors choose to ligate only these 10 widely patent PV and, in these other 217 testes, only low scrotal orchiopexy was performed. In a median follow-up of 44 months (range 20-73), only one complication in 'no widely patent PV' group (wound infection) and one complication (reascending testis) in 'widely patent PV' group occurred. As the authors mentioned in their paper, two major concerns with ligation of PV are to obtain a sufficient cord length for appropriate orcihopexy and prevent an inguinal hernia. During their follow-up, there were no reascending testis or hernia occurred in 'non-ligated PV' group. In conclusion, low scrotal approach ochiopexy is a safe procedure for patients with prescrotal crytorchidism and ligation of PV is not absolutely indicated when the PV is not widely patent.

Tukut Doğanca, MD

\section{Re: Factors Predicting Outcomes of Micropercutaneous Nephrolithotomy: Results from a Large Single-Centre Experience}

\author{
Ganpule A, Chhabra JS, Kore V, Mishra S, Sabnis R, Desai M \\ Muljibhai Patel Urological Hospital, Gujarat, India
}

BJU lnt 2016;117:478-483. doi: 10.1111/bju.13263. Epub 2015 Sep 30.

\section{EDITORIAL COMMENT}

The recently developed micropercutaneous nephrolithotomy (microperc) is the miniaturized version of mini-percutaneous nephrolithotomy (PCNL) and standardPCNL that allows for safe access and stone disintegration under direct vision. In this single-centre study, the authors aimed to define the role of microperc in the management of renal calculi and analyse factors predicting outcomes. A total of 139 patients, who underwent microperc between June 2010 and November 2014, were enrolled in this study. Microperc was successful in 119 (91.53\%) patients, while in 11 patients (8.46\%) some residual fragments were seen on imaging. Of the total study group, conversion to mini- or standard PCNL was required in nine patients (6.47\%). Overall complication rate of $11.53 \%$, primarily minor complications comprising renal colic and urinary tract infection. On multivariate analysis, stone density (HU), stone number and stone volume were significantly associated with the dependent variable stone clearance. Multivariate analysis showed that intra-operative complications and stone number were significantly associated with conversion to mini- or standard PCNL. Stone volume threshold of $1.000 \mathrm{~mm}^{3}$ was a significant predictor of stone clearance in univariate and multivariate analyses, regardless of the stone location.

Some limitations of this study, relatively small sample size and particularly the retrospective and non-comparative design, should be highlighted. EAU guidelines recommend shockwave lithotripsy (SWL) or retrograde intrarenal surgery as the primary treatment modality for stones $<10 \mathrm{~mm}$ in size. Although microperc is presently being used for small to moderate stones, the very indication that holds for SWL as well, it is notable that SWL is influenced by stone location and pelvicalyceal anatomy, and may require multiple sessions. Nevertheless, microperc has an inherent limitation in that the stone fragments cannot be retrieved for analysis. It has been noted that the closed system leads to a pressure rise, especially in scenarios of impacted pelvic stones and longer operating times. This problem may be even more serious in pediatric patients. 